Associate editor: B. Teicher

\title{
Targeting DNA damage response: Threshold, chromatin landscape and beyond
}

\author{
Stefania Gonfloni * \\ Department of Biology, University of Rome “Tor Vergata”, via della Ricerca Scientifica, I-00133 Rome, Italy
}

\section{A R T I C L E I N F O}

\section{Keywords:}

DNA damage

Histone modifications

DNA repair

$\gamma-\mathrm{H} 2 \mathrm{AX}$

Chromatin

Tip60

c-Abl

ATM

\begin{abstract}
A B S T R A C T
Cells are continually exposed to DNA assaults from exogenous and endogenous sources. To maintain genomic integrity, cells have evolved a highly conserved mechanism for repairing DNA lesions and, in particular, DNA double strand breaks (DSBs). Emerging evidence indicates that DNA repair/signaling machinery acts in an integrated fashion with chromatin structure at damaged sites. This review focuses on the interplay between histone modifications and the chromatin-mediated response to DNA damage.
\end{abstract}

(C) 2013 Elsevier Inc. All rights reserved.

\section{Contents}

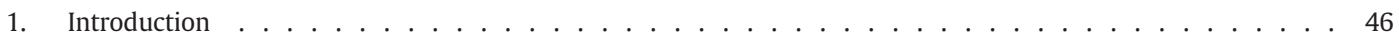

2. When does DNA damage occur? . . . . . . . . . . . . . . . . . . . . . . . . . . 47

3. How do cells respond to DNA insults? . . . . . . . . . . . . . . . . . . . . . . . . . 47

4. Stepwise response induced by DNA lesions . . . . . . . . . . . . . . . . . . . . . . . . . . . . . . . 47

5. Dynamics of histone modifications influences chromatin structure . . . . . . . . . . . . . . . . . . . . . . 48

6. Timing and threshold for DDR . . . . . . . . . . . . . . . . . . . . . . . . . . . . . . . . . . 48

7. Connections between DNA damage signaling and chromatin landscape . . . . . . . . . . . . . . . . . . . . 49

8. Concluding remarks . . . . . . . . . . . . . . . . . . . . . . . . . 50

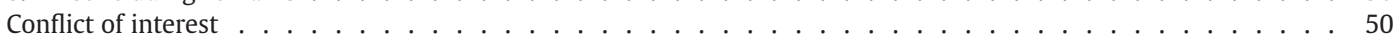

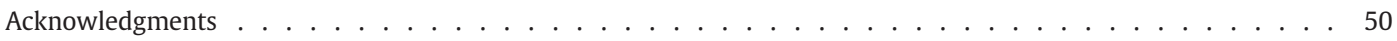

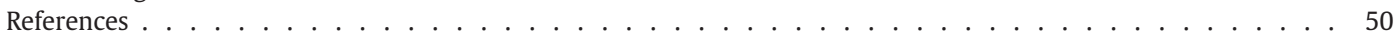

\footnotetext{
Abbreviations: DSBs, double strand breaks; $\gamma$-H2AX, phosphorylation of histone variant $\mathrm{H} 2 \mathrm{AX}$ in Ser139; SUMO, small ubiquitin modifier; ROS, reactive oxygen species; IR, ionizing radiation; Tip60, Tat-interactive 60; HAT, histone acetyltransferase; HDAC, histone deacetylase; NHEJ, non homologous end joining; HR, homologous recombination; DDR, DNA Damage response; NER, nucleotide excision repair; BER, base excision repair; MMR, mismatch repair; HP1, heteochromatin Protein1; INO80, inositol requiring 80; SWR1, sick with Rat8 ts; FHA, forkhead-associated; BRCT, breast cancer-terminal; UBD, ubiquitin binding domain; SIM, sumo interacting motif; MDC1, mediator of DNA damage checkpoint 1; BRCA1, breast cancer 1, early onset; FACT, facilitates chromatin transcription; SIRT1, sirtuin (silent mating type information regulation 2 homolog)1 deacetylase; WSTF, WilliamsBeuren syndrome transcription factor tyrosine kinase; EYA, eyes absent protein phosphatase; MOF, males absent on the first; ATM, ataxia telangiectasia; MRN, Mre11/ Nbs1/Rad50 complex; RNF8, RING finger protein 8; UBC13, ubiquitin-conjugating enzyme E2 13.

* Tel.: +3906 72594319; fax: +3906 2023500

E-mail address: Stefania.Gonfloni@uniroma2.it.
}

\section{Introduction}

Maintaining genomic integrity in response to DNA assaults is an essential process for living cells (Jackson \& Bartek, 2009; Ciccia \& Elledge, 2010; Negrini et al., 2010). To achieve this goal, cells use dynamic signaling networks that can sense, interpret and respond to various DNA stressors (Bekker-Jensen et al., 2010). Signal duration and amplitude of stresses evoke very complex patterns of proteinprotein interactions. Canonical DNA damage kinases trigger several posttranslational modifications of DNA signaling proteins (Al-Hakim et al., 2010; Bensimon et al., 2011). These modifications show a dynamic behavior where signals of phosphorylation are interpreted through ubiquitin- (or Sumo-) mediated signal decoding (van Attikum \& Gasser, 2005; Ramaekers \& Wouters, 2011). The consequence is a timely recruitment (and disassembly) of large complexes near the damaged site (Panier \& Durocher, 2009; van Attikum \& Gasser, 2009; Ulrich \& Walden, 2010). The first modification induced by DNA damage affects the histone variant $\mathrm{H} 2 \mathrm{AX}$ on S139 to form $\gamma$-H2AX. S139- 
phosphorylation induces a massive accumulation of proteins in $\gamma$-H2AX foci (Stucki et al., 2005). The latter are microscopically visible aggregates, present in large segments of chromatin flanking the lesions, where the balance of opposing enzymes drives targeted recruitment, proteinprotein interactions and posttranslational modifications (Huen et al., 2007; Kolas et al., 2007; Mailand et al., 2007; Wang \& Elledge, 2007; Doil et al., 2009; Stewart et al., 2009). Most of these enzymes are highly connected "hub" proteins interacting in complex regulatory circuits that allow temporary local clustering and reversibility of the interactions (Maiani et al., 2011). A second layer of complexity of DNA damage signaling relies on its profound impact on chromatin status (Gasch et al., 2001; Rieger \& Chu, 2004; Reinhardt et al., 2011). At first glance, the DNA damage response induces a global repression of transcription (Vichi et al., 1997; Svejstrup, 2002) followed by a delayed transcriptional response, prolonging cell cycle arrest (Fei \& El-Deiry, 2003; Elkon et al., 2005). In recent years, posttranscriptional control circuits are emerging as a third level of regulation of DDR signaling networks, recently reviewed by Boucas and co-workers (Boucas et al., 2012). They involve either RNA-binding proteins (RBPs) or non-coding RNAs, each of them impacting on protein biosynthesis (Fan et al., 2002; Matsuoka et al., 2007; Paulsen et al., 2009; Francia et al., 2012).

Chromatin structure and histone modifications are actively interconnected elements controlling the mechanisms underlying genome-integrity maintenance. While it appears intuitive that chromatin compaction protects DNA from lesions, chromatin state plays a central role for local signaling at the break points. For instance, "open" relaxed chromatin domains influence loading of DNA repair proteins onto chromatin near the site of the lesion (Xu \& Price, 2011). Remodeling protein complexes (ATPase and Tip60 acetyl transferase HAT) can modulate chromatin accessibility and in combination with histone modifications promote subsequent chromatin ubiquitination (Lukas, 2010; Shanbhag et al., 2010). Thus, the dynamic landscape of chromatin, through a sophisticated combination of posttranslational modifications, may directly influence the choice of a specific DNA repair pathway adopted by the cells (Xu \& Price, 2011; Chapman et al., 2012; Soria et al., 2012). In this review, I will focus specifically on the interplay between histone modifications, centered on H2AX phosphorylation, and the chromatin-mediated response to DNA damage.

\section{When does DNA damage occur?}

The major endogenous sources of DNA damage are reactive oxygen species (ROS) or unrepaired DNA lesions causing replication fork collapse in the cell (Ward \& Chen, 2001; Zou \& Elledge, 2003; Kryston et al., 2011; De Zio et al., 2012). DNA breaks also arise following treatment with exogenous genotoxic agents or ionizing radiation (IR) (Roos \& Kaina, 2012). To counteract these different types of lesions cells explore multiple DNA repair pathways (Aziz et al., 2012). One type of lesion, the DNA Double strand break (DSB) is particularly dangerous for cells, as free DNA ends created by the lesion are susceptible to degradation or re-ligation, and promote genomic instability. DSBs can be generated by collapse of replication fork, ionizing radiation IR, exposure of specific compounds, and even during the processing of other lesions (Aziz et al., 2012). Under normal circumstances, DSBs are even programmed by the cell: in germ cells during meiotic repair (Keeney \& Neale, 2006), or in lymphocytes during rearrangement of immunoglobulin genes (Dudley et al., 2005). DSBs can be repaired by two major pathways, homologous recombination (HR) and non homologous end-joining (NHEJ). In NHEJ, the broken ends are directly ligated in an error-prone manner (Lieber, 2010), frequently causing small insertions, deletions or substitutions at the break site. On the contrary, HR requires DNA end processing and a template (homologous DNA molecule) for error-free copying and repairing of the lesion.

\section{How do cells respond to DNA insults?}

Many studies done in the past on DDR have defined a hierarchical order among the different players, those, depending on their subcellular localization, are described as sensors, transducers, mediators and effectors (Bekker-Jensen et al., 2010). Sensors promptly respond to signals and are directly bound to chromatin, whereas transducers, mediators and effectors guide the DNA damage response events (Jackson \& Bartek, 2009; Ciccia \& Elledge, 2010; Negrini et al., 2010). Intuitively, a simple activation and regulation of this cascade occurs when DNA damage is not extensive and can be promptly repaired. When damage is prolonged, the balance of multi-layered connected pathways for DNA repairing and cell cycle arrest or initiating apoptosis (or senescence), leads cells to a decision point between survival or death (Ciccia \& Elledge, 2010). DNA repair is essential for cell viability and normal growth, while irreparable damage definitely leads to a programmed cell death. To counter this, cells explore several different mechanisms for repairing DNA breaks. According to the type of damage, they use nucleotide excision repair (NER), base excision repair (BER), mismatch repair (MMR). In addition, to repair the most dangerous lesions as DNA double strand breaks (DSB), cells can use two distinct pathways the (non-homologous end joining) NHEJ or HR (homologous recombination) repair systems (Aziz et al., 2012). Both compete one with each other and the choice between them is linked to the cell cycle phase and to the accumulation of specific markers. Many studies in the past have indicated that NHEJ pathway mainly occurs during G1-early $\mathrm{S}$ phase, even if it can be used during the whole cell cycle. On the contrary, HR repair is only used in late S-G2 phase (Takata et al., 1998; Shrivastav et al., 2008).

\section{Stepwise response induced by DNA lesions}

Emerging evidence suggests an active role of chromatin in DNA damage response (Bao, 2011; Luijsterburg \& van Attikum, 2011; Lukas et al., 2011; Xu \& Price, 2011; Miller \& Jackson, 2012; Soria et al., 2012). Chromatin is a complex scaffold that compacts and organizes DNA in eukaryotic cells. Chromatin structure relies on a basic unit, the nucleosome. The nucleosome is formed by 146 base pair of DNA wrapped around a core composed by four different histones H2A, H2B, H3 and H4 (Campos \& Reinberg, 2009). The central core of the nucleosome is formed by two H3-H4 dimers, surrounded by two H2A-H2B dimers (Campos \& Reinberg, 2009). Chromatin structure is quite dynamic and can be modified through different mechanisms. Diverse classes of enzymes can modulate chromatin compaction. One class consists of large multi-protein complexes that need the energy of ATP hydrolysis to slide the nucleosomes or alter/ exchange histone composition within the chromatin fibers. Another class of enzymes mediates covalent modifications of histone tails. Histone tails extend outward from the nucleosome and contain sites for regulatory modifications such as phosphorylation, ubiquitylation, methylation and acetylation. Histone modifications regulate chromatin functions. For instance, Lysine methylation (K4) of N-terminal tail of histone H3 (H3K4me) leads to gene activation and euchromatin formation (Margueron \& Reinberg, 2010). On the contrary, methylation of histone H3 on K9 (H3K9me) creates an interaction motif for HP1 (Heterochromatin Protein 1) proteins, and promotes the formation of heterochromatin, leading to gene silencing (Bannister et al., 2001; Grewal \& Jia, 2007). Changes in histone modification, implicated in the switch from euchromatin to heterochromatin, are regulated by pair-opposing enzymes that allow the dynamic rewriting of histone marks as histone acetyl transferases (HATs) and histone deacetylases (HDACs), HATs/HDACs, and (histone methyltransferase (HTMs) and histone demethylases (HDMs) HTMs/HDMs, also by DNA methyltransferase (Gallinari et al., 2007). Histone modifications, when present in specific combinations with other histone marks, can form a specific 'code' for recruiting some important effectors required for signaling 
amplification and/or chromatin remodeling (Downs et al., 2004; Morrison et al., 2004; van Attikum et al., 2004). Often, some histone marks compete one with each other or even for the same residue. This is particularly relevant for lysine residue, which can be alternatively modified by ubiquitylation, sumoylation, acetylation or methylation. Posttranslational modifications of histone by changing the charge of amino acid residue can alter the stability of the interaction with DNA or with other charged protein interfaces (Cairns, 2005; Kouzarides, 2007; Shahbazian \& Grunstein, 2007; Campos \& Reinberg, 2009; Suganuma \& Workman, 2011).

In addition, histone modifications alter nucleosome composition, promoting the release of phospho-H2A histone from damaged chromatin (Ikura et al., 2007), through a histone variant exchange event. This event is regulated by an opposing action of two INO80 and SWR1 remodelers (Papamichos-Chronakis et al., 2006). Interestingly, enrichment of certain histone variants (e.g. H2AX) localizes sparse specific domains within chromatin fiber. Thus, differential expression of histone variants throughout the cell cycle and their respective positioning/localization onto chromatin (Talbert \& Henikoff, 2010; Boyarchuk et al., 2011; Szenker et al., 2011) critically influence DNA damage response. In addition, histone chaperones (in synergy with ATP-dependent remodeling factors) (Clapier \& Cairns, 2009) regulate the exchange with free unlabeled histones acting as "erasers" for specific posttranslational histone modifications. In a wide sense, histone variants (with their posttranslational labels) could be considered a "removable/temporary" platform for assembling local signaling circuitry onto chromatin. The amplitude and duration of such circuitry is likely regulated by feedback control and contribute to the fine-tuning of the DDR and to the maintenance of genome integrity.

\section{Dynamics of histone modifications influences chromatin structure}

Chromatin packaging is variable, typically distinguished in two states: a condensed (heterochromatin) and a more open (euchromatin) structure. Euchromatin is an accessible, gene rich and transcriptionally active region. Histones within euchromatin are highly acetylated and $\mathrm{H} 3$ is methylated on $\mathrm{K} 4$ and $\mathrm{K} 36$ (H3K4me and H3K36me2) (Barski et al., 2007; Guenther et al., 2007). On the contrary, heterochromatin has low gene density and instead contains many repetitive sequences (de Wit et al., 2007; Peng \& Karpen, 2008). Histones within heterochromatin are poorly acetylated and $\mathrm{H} 3$ is highly methylated on $\mathrm{K} 9$ and $\mathrm{K} 36$ (H3K9me3; H3K36me3) (Pokholok et al., 2005; Vakoc et al., 2006; Guenther et al., 2007; Peng \& Karpen, 2008). Posttranslational modifications of histones (PTM) can act as binding motif for proteins containing PTM-binding domain (Yun et al., 2011). For instance, bromodomains bind specifically to acetylated lysine residues, whereas chromodomains recognize methylated lysine residues (Kouzarides, 2007; Ruthenburg et al., 2007; Shahbazian \& Grunstein, 2007). Other important PTM-binding domains present in DDR proteins include FHA or BRCT (breast cancer1, early-onset) domains (Mohammad \& Yaffe, 2009) that recognize phosphorylated epitopes on target proteins as well as UBD (ubiquitin binding domain) domains and SIM (sumo interacting motif) motifs that bind ubiquitin and SUMO respectively (Kerscher et al., 2006; Hofmann, 2009).

The first evidence that chromatin is modified at site of DNA break came from the discovery that the histone variant $\mathrm{H} 2 \mathrm{AX}$ is phosphorylated on its C-terminal tail following DNA damage (Miller \& Jackson, 2012). Exposure of cells to DNA damaging agents activates phosphorylation of many target proteins (Bensimon et al., 2011). However, S139-phosphorylation of histone variant H2AX is considered one of the early markers of the DDR. This modification modulates the H2AX-interaction with DNA and promotes assembly of signaling complexes onto chromatin. While, there is a little evidence that $\gamma$-HAX has been involved in DNA repair per se, it plays a central role for the initiation and amplification of DNA damage signals.
Signaling at DSBs induces $\gamma$-H2AX, eliciting timely engagement of MDC1 (mediator of DNA damage checkpoint 1), 53BP1 (p53 binding protein 1) and BRCA1 (breast cancer 1, early onset). Bidirectional spreading of $\gamma$-H2AX far from DNA lesions further enhances damage signaling and helps to delineate the chromatin region involved in DDR (Yuan et al., 2010). Silenced chromatin regions are not permissive for $\gamma$-H2AX spreading (Kim et al., 2007) and H2AX dynamics within the nucleosome are coupled to its various posttranslational modifications. Besides phosphorylation, other modifications such as ubiquitylation and acetylation of H2AX variant promote the recruitment of DDR proteins near the breaks. DNA damage-dependent modifications (i.e. poly ADP-rybosylation) of histone chaperone FACT (Facilitates Chromatin Transcription) inhibit its interaction with nucleosomes reducing the H2AX/H2A exchange (Du et al., 2006; Heo et al., 2008). On the contrary, the Tip60 complex (Tat-interactive protein 60 ), is a chromatin modifier and acetylates $\mathrm{H} 2 \mathrm{AX}$, enhancing its mobility within chromatin (Ikura et al., 2007). Interestingly Tip60-mediated acetylation (on K5) is required for the subsequent ubiquitination of H2AX (on K119) by RNF8/UBC13 (ubiquitin-conjugating enzymes) (see Fig. 1) (Ikura et al., 2007). In mammalian cells, SIRT1 (a protein deacetylase) negatively regulates Tip60-mediated acetylation of histone H2AX (Yamagata \& Kitabayashi, 2009). Conversely, in yeast, a remodeling factor INO80 (Inositol requiring 80) retains phospho-H2A within the nucleosome (PapamichosChronakis et al., 2006). In combination with remodeling factors that promote $\gamma$-H2AX eviction from chromatin, several protein phosphatases can also negatively regulate the function of $\gamma$-H2AX by promoting its dephosphorylation (Nazarov et al., 2003; Chowdhury et al., 2005; Keogh et al., 2006; Chowdhury et al., 2008). The histone variant H2AX is also decorated by additional modifications which contribute to chromatin response to DNA damage (reviewed by Miller \& Jackson, 2012). H2AX is phosphorylated on its C-terminal tyrosine residue by WSTF, a non canonical tyrosine kinase (Xiao et al., 2009). Following DNA damage, this phoshorylation is removed by the phosphatase EYA (Cook et al., 2009). The pair-opposing enzymes WSTF or EYA are both important for an effective DDR, pointing out the relevance of this tyrosine modification for H2AX function (Cook et al., 2009; Xiao et al., 2009). In sum, the fine-tuning of $\mathrm{H} 2 \mathrm{AX}$ modifications and its dynamics mediated by the effect of specific action of pair enzymes offers a tunable switch for DNA damage signaling events.

\section{Timing and threshold for DDR}

Exposure of cells to genotoxic compounds activates the phosphatidylinositol-3-kinase-related kinase (PI3KK) family of kinases (ATM, ATR and DNA-PKcs). Although the PI3K related kinases are considered important players in DDR, an unrelated tyrosine kinase c-Abl has more recently also associated with the activation of key upstream event of DDR (Gonfloni, 2010a; Meltser et al., 2011; Wang et al., 2011; Maiani et al., 2012). In response to DNA damage, PI3K related kinases mediate the phosphorylation of H2AX on S139 $(\gamma-\mathrm{H} 2 \mathrm{AX})$. However, other numerous modifications (acetylation and methylation) occur on core histones in response to DNA damage. Two key modifications occur on H4, K16 acetylation and K20 methylation respectively. H4K16Ac modification is mediated by Tip60 and MOF (Murr et al., 2006; Li et al., 2010). While Tip60 mediates H2A and H4K16 acetylation at the site of break (Fig. 1), MOF seems to control global level of H4K16ac and does not localize at DSB (Sharma et al., 2010). Interestingly, Tip60 can also be activated by a histone mark (H3K9me3) associated with heterochromatin (Sun et al., 2009; Sun et al., 2010). Lack of either HAT enzymes (Tip60 and MOF) causes defective HR and NHEJ repair, suggesting that both are required for efficient DSB repair. However, the precise mechanism by which acetylation of H4K16 promotes DNA repair still remains elusive. Interestingly, a combination of histone marks such as $\mathrm{H} 2 \mathrm{~b}-\mathrm{Ub}$ (ubiquitilated H2B) and H4K16ac induces decompaction of nucleosome (Shogren-Knaak \& Peterson, 2006; Fierz et al., 2011). Thus, 

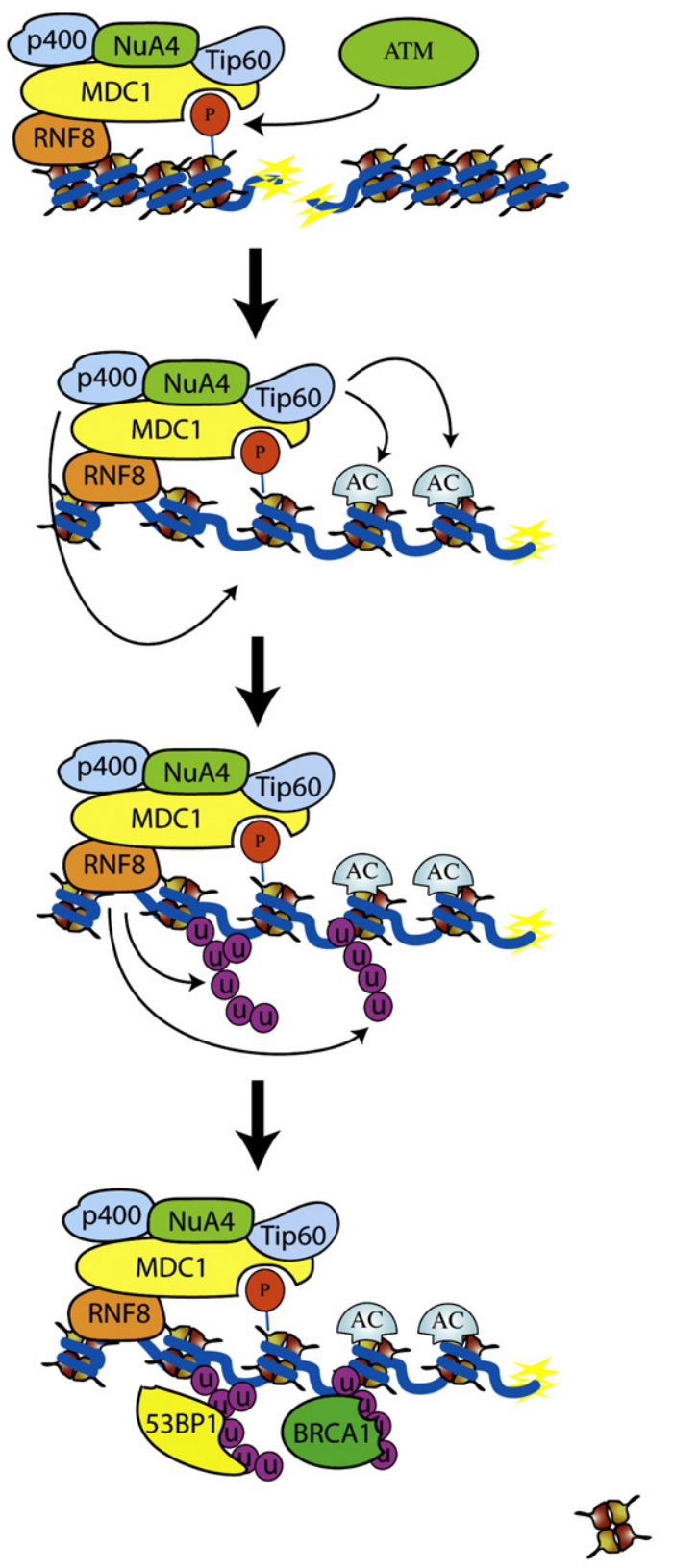

nucleosome

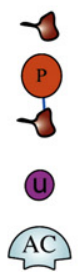

histone

$\gamma \mathrm{H} 2 \mathrm{AX}$

ubiquitin

acetylation

Fig. 1. Tip60-mediated acetylation of $H 2 A X$ promotes Ubiquitin-dependent signaling at damage sites. H2AX phosphorylation by ATM provides a docking site for MCD1 and leads to the recruitment of ubiquitin ligase RNF8 and NuA4 complex at damaged sites. (NuA4 is a large complex form by Tip60, p400 motor ATPase and other subunits). Then, Tip60 mediates acetylation of histone H2AX, in combination with the action p400 ATPase, generates an open relaxed chromatin structure, facilitating ubiquitindependent signaling at the damage sites. $A c=$ acetylation, $\mathrm{p}=$ phosphorylation.

Tip60-mediated acetylation at DSB promotes instability of nucleosomes near the site of break. This could facilitate a shift of the local chromatin structure into an open relaxed conformation more permissive for the ubiquitin-dependent signaling at the damage site.
Posttranslational modifications of histones, both in the tails and in the core region affect the functional landscape of chromatin by regulating DNA accessibility. A key aspect of the role of histone modifications relies on their dynamic nature (Krebs, 2007); the precise timing of addition and removal of specific marks (or entire histone) determines a dynamic temporal regulation of chromatin functions (Krebs, 2007). H2AX-phosphorylation on S139 ( $\gamma$-H2AX) is an early marker of DDR. Several studies indicate that $\gamma-\mathrm{H} 2 \mathrm{AX}$ acts as beacon for proteins with dedicated phosphor-S/T binding domains (FHA, BRCT), promoting a sequential assembly of ubiquitin-dependent signaling cascades (see Fig. 1). Thus, DNA breaks initially promote repair and also a DNA signaling cascade for assisting repair (Yuan \& Chen, 2010). At damaged site, the efficiency of signaling is enhanced by local concentration of factors. Signaling amplitude and duration are regulated through dynamic editing and removal of specific marks. This eventually could arrest the repair process for an alternative path leading to cell death. Most likely, survival of DNA-damaged cells strictly depends both on the removal of the lesion coupled with an efficient DNA damage signaling decay. This aspect is particularly crucial in the cellular context of perinatal oocytes more sensitive to genotoxic stress than somatic cells. (Gonfloni, 2010a; Gonfloni, 2010b; Maiani et al., 2012). In immature oocytes, pharmacological inhibition of c-Abl tyrosine kinase attenuates the toxic effect induced by chemotherapeutic drugs (Gonfloni et al., 2009; Maiani et al., 2012). Our studies indicate that c-Abl inhibition works on distinct levels of DNA damage signaling both at early time points reducing on $\gamma-\mathrm{H} 2 \mathrm{AX}$ phosphorylation and then impinging on a downstream effector TAp63 (Gonfloni et al., 2009; Maiani et al., 2012). This supports the hypothesis that amplification of DNA damage signaling cascade leads germ cells towards death, as a default path, if not attenuated (Maiani et al., 2011).

\section{Connections between DNA damage signaling and chromatin landscape}

Now we move to the next point, how the H2AX dynamics, regulated by posttranslational modifications, in tandem with histone chaperones and remodelers contribute to the DDR? Recent evidence supports an active role of chromatin in DNA damage response. Chromatin compaction protects DNA from lesions. Heterochromatin, compared to euchromatin, is densely compact, transcriptionally silent and may act as barrier limiting access to all DDR factors (Soria et al., 2012). However, generation of DNA breaks and the early steps of DNA damage signaling and repair occur efficiently within the heterochromatin domains (Baldeyron et al., 2011; Chiolo et al., 2011; Jakob et al., 2011). Final steps of DNA signaling and repair (accumulation of RAD 51 and $\gamma$-H2AX spreading) are instead relocalized and confined in more accessible environment at the periphery of the heterochromatin region. The expansion of the heterochromatin facilitates the repositioning of damaged DNA near the surrounding euchromatin domains (Chiolo et al., 2011; Jakob et al., 2011; Baldeyron et al., 2011) to finalize late steps of DNA repair (Soria et al., 2012). Interestingly, a similar repositioning is observed during DNA replication (Quivy et al., 2004). It is possible that cells have evolved such mechanisms to prevent ectopic recombination between the repetitive sequences within heterochromatin, by restricting the processing of DNA ends at the periphery of heterochromatin (Quivy et al., 2004; Peng \& Karpen, 2008; Chiolo et al., 2011). This could in turn prevent possible chromosomal rearrangements and genomic instability. How a sophisticated control on DNA accessibility is linked to DNA repair? ATM is a master regulator of DNA damage response. ATM mediates the phosphorylation of $\gamma$-H2AX promoting a signaling cascade, which leads to the assembly of DNA repair machinery and to activation of cell cycle checkpoints. However, how DNA breaks upregulate the activity of ATM is not completely clarified. Recent biochemical studies indicate that the autophosphorylation of ATM on S1981 is not the primary mechanism for ATM activation. This autophosphorylation is indeed dispensabile for 
ATM function under some conditions (Lee \& Paull, 2005; Dupre et al., 2006; Pellegrini et al., 2006). Most likely ATM activation is also mediated through binding with MRN (complex formed by mre11 nuclease, Rad50 ATPse and Nbs1) DNA binding complex (Uziel et al., 2003; Difilippantonio et al., 2005; Falck et al., 2005; Lee \& Paull, 2005; Cerosaletti et al., 2006). Deletion of mre11, rad50 or nbs1 elements of MRN complex significantly reduces activation of ATM following DNA damage in vivo (Uziel et al., 2003; Difilippantonio et al., 2005; Falck et al., 2005; Cerosaletti et al., 2006). Recent studies indicate that Tip60 acetyltransferase is required for ATM activation (Sun et al., 2005). Tip60 and ATM form a complex in which Tip60 interacts with highly conserved FATC domain of ATM. This interaction in turn facilitates the acetylation of ATM on K3016 (Jiang et al., 2006; Sun et al., 2007). Tip60's chromodomain recognizes specifically H3K9me3 (Sun et al., 2009); and the binding with H3K9me3 increases Tip60 HAT activity through an allosteric mechanism. Mutations in the chromodomain binding motif prevent both the interaction between Tip60 and H3K9me3 and the upregulation of Tip60's HAT activity. The consequence
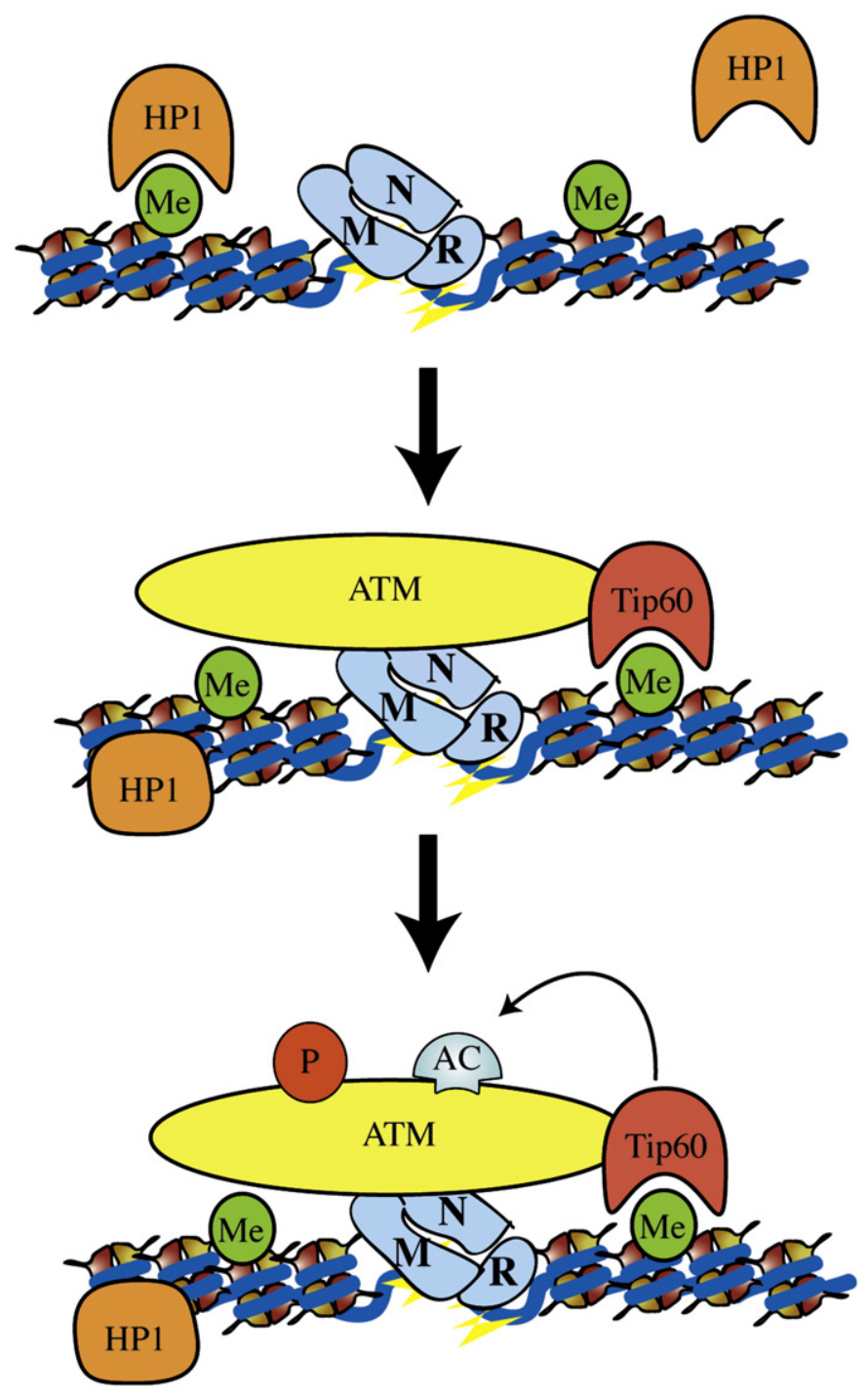

Fig. 2. Early steps of DNA damage response: a potential model for ATM activation by Tip60. Following DNA damage, MRN is recruited to DSB. In parallel, HP1 proteins are released from $\mathrm{H} 3 \mathrm{~K} 9 \mathrm{me} 3$ (heterochromatin histone mark). MRN promotes targeted recruitment of the inactive ATM-Tip60 complex at DSBs. This event facilitates the interaction between the chromodomain of Tip60 and H3K9me3, enhancing Tip60's HAT activity through an allosteric mechanism. Interaction between MRN and Abl, together with acetylation of ATM mediated by Tip60, activates the kinase activity of ATM. $\mathrm{Me}=$ methylation, $\mathrm{Ac}=$ acetylation, $\mathrm{p}=$ phosphorylation . is a reduction of acetylation and activation of ATM kinase activity mediated by Tip60 (Sun et al., 2009). Reduction of global H3K9me3 levels, by acting on opposing enzymes either on KDM4D demethylases (Whetstine et al., 2006) or Suv39 h1 and Suv39hu2 methyltransferase (Peters et al., 2001), significantly decreases Tip60 activation following DNA damage (Sun et al., 2009). In addition, cells with low level of H3K9me3 show an increased sensitivity to IR and genomic instability (Sun et al., 2009). Taken together, these observations suggest that the chromodomain functions as sensor for Tip60 activation, modulating both recruitment and HAT activity at DNA damage sites. Thus, a direct interaction between methylated histones and Tip60's chromodomain indicates that chromatin structure plays a role in DNA repair (Sun et al., 2010). Tip60 is stably associated with ATM in cells, and both proteins are recruited at DSBs (Fig. 2), most likely in an inactive state (Sun et al., 2005; Jiang et al., 2006). Recent evidence indicates that loss of functional MRN complex delayed the recruitment and activation of Tip60 after DNA damage (Sun et al., 2009). However the requirement of both MNR complex and Tip60 for ATM activation in vivo remains still elusive as well as the role of other potential effectors associated in the complex. Recent evidence indicates that Tip60 interacts with c-Abl tyrosine kinase both in vitro and in vivo and is an upstream c-Abl modifier in response to DNA damage. Interestingly, c-Abl acetylation mediated by Tip60 required an ATM-mediated phosphorylation of c-Abl on S465 (Jiang et al., 2011).

\section{Concluding remarks}

In conclusion, chromatin is emerging as an integral player in the DDR (Soria et al., 2012). Cells have evolved dedicated signaling and repair machinery to control the chromatin structure facilitating (or preventing) DNA access in a dynamic way at the site of damage and nearby. This sophisticated machinery includes enzymes involved in posttranslational modifications of histones, incorporation of histone variants and ATP-dependent chromatin remodeling. Emerging evidence indicates that all these three classes of components are direct players in DNA damage response induced by DSBs acting in an integrated fashion. Histone deacetylases (HDACs) promote chromatin condensation and are considered promising targets for cancer therapy because their inhibition is preferentially toxic for some cancer cells (Johnson et al., 2002; Minucci \& Pelicci, 2006). More sophisticated techniques based on ChIP assay and the development of powerful DSD-inducing systems are rapidly improving our understanding of DSB repair processes (Polo \& Jackson, 2011). Undoubtedly, this will provide new hints for the development of targeted therapies for DDR in a global integrated fashion.

\section{Conflict of interest}

The author declares no conflict of interest.

\section{Acknowledgments}

The author wishes to apologize to those colleagues whose significant studies were not cited due to space limitations. The author wishes to thank Emiliano Maiani for assistance with the figure, Gianni Cesareni and Marc Diederich for support. This work is supported by funds provided by AIRC (Associazione Italiana Ricerca sul Cancro) and Televie (Luxembourg).

\section{References}

Al-Hakim, A., Escribano-Diaz, C., Landry, M. C., O'Donnell, L., Panier, S., Szilard, R. K., et al. (2010). The ubiquitous role of ubiquitin in the DNA damage response. DNA Repair (Amst) 9, 1229-1240.

Aziz, K., Nowsheen, S., Pantelias, G., Iliakis, G., Gorgoulis, V. G., \& Georgakilas, A. G. (2012). Targeting DNA damage and repair: embracing the pharmacological era for successful cancer therapy. Pharmacol Ther 133, 334-350. 
Baldeyron, C., Soria, G., Roche, D., Cook, A. J., \& Almouzni, G. (2011). HP1alpha recruitment to DNA damage by p150CAF-1 promotes homologous recombination repair. $J$ Cell Biol 193, 81-95.

Bannister, A. J., Zegerman, P., Partridge, J. F., Miska, E. A., Thomas, J. O., Allshire, R. C., et al. (2001). Selective recognition of methylated lysine 9 on histone $\mathrm{H} 3$ by the HP1 chromo domain. Nature 410, 120-124.

Bao, Y. (2011). Chromatin response to DNA double-strand break damage. Epigenomics 3, 307-321.

Barski, A., Cuddapah, S., Cui, K., Roh, T. Y., Schones, D. E., Wang, Z., et al. (2007). High-resolution profiling of histone methylations in the human genome. Cell 129, 823-837.

Bekker-Jensen, S., Rendtlew Danielsen, J., Fugger, K., Gromova, I., Nerstedt, A., Lukas, C., et al. (2010). HERC2 coordinates ubiquitin-dependent assembly of DNA repair factors on damaged chromosomes. Nat Cell Biol 12, 80-86 (sup pp 81-12).

Bensimon, A., Aebersold, R., \& Shiloh, Y. (2011). Beyond ATM: the protein kinase landscape of the DNA damage response. FEBS Lett 585, 1625-1639.

Boucas, J., Riabinska, A., Jokic, M., Herter-Sprie, G. S., Chen, S., Hopker, K., et al. (2012) Posttranscriptional regulation of gene expression-adding another layer of complexity to the DNA damage response. Front Genet 3, 159.

Boyarchuk, E., Montes de Oca, R., \& Almouzni, G. (2011). Cell cycle dynamics of histone variants at the centromere, a model for chromosomal landmarks. Curr Opin Cell Biol 23, 266-276.

Cairns, B. R. (2005). Chromatin remodeling complexes: strength in diversity, precision through specialization. Curr Opin Genet Dev 15, 185-190.

Campos, E. I., \& Reinberg, D. (2009). Histones: annotating chromatin. Annu Rev Genet 43, 559-599.

Cerosaletti, K., Wright, J., \& Concannon, P. (2006). Active role for nibrin in the kinetics of ATM activation. Mol Cell Biol 26, 1691-1699.

Chapman, J. R., Sossick, A. J., Boulton, S. J., \& Jackson, S. P. (2012). BRCA1-associated exclusion of 53BP1 from DNA damage sites underlies temporal control of DNA repair.J Cell Sci $125,3529-3534$

Chiolo, I., Minoda, A., Colmenares, S. U., Polyzos, A., Costes, S. V., \& Karpen, G. H. (2011) Double-strand breaks in heterochromatin move outside of a dynamic HP1a domain to complete recombinational repair. Cell 144, 732-744.

Chowdhury, D., Keogh, M. C., Ishii, H., Peterson, C. L., Buratowski, S., \& Lieberman, J. (2005). gamma-H2AX dephosphorylation by protein phosphatase $2 \mathrm{~A}$ facilitates DNA double-strand break repair. Mol Cell 20, 801-809.

Chowdhury, D., Xu, X., Zhong, X., Ahmed, F., Zhong, J., Liao, J., et al. (2008). A PP4-phosphatase complex dephosphorylates gamma-H2AX generated during DNA replication. Mol Cell 31, 33-46.

Ciccia, A., \& Elledge, S. J. (2010). The DNA damage response: Making it safe to play with knives. Mol Cell 40, 179-204.

Clapier, C. R., \& Cairns, B. R. (2009). The biology of chromatin remodeling complexes. Annu Rev Biochem 78, 273-304.

Cook, P. J., Ju, B. G., Telese, F., Wang, X., Glass, C. K., \& Rosenfeld, M. G. (2009). Tyrosine dephosphorylation of H2AX modulates apoptosis and survival decisions. Nature $458,591-596$

de Wit, E., Greil, F., \& van Steensel, B. (2007). High-resolution mapping reveals links of HP1 with active and inactive chromatin components. PLoS Genet 3, e38.

De Zio, D., Bordi, M., \& Cecconi, F. (2012). Oxidative DNA damage in neurons: Implication of $\mathrm{ku}$ in neuronal homeostasis and survival. Int J Cell Biol 2012, 752420.

Difilippantonio, S., Celeste, A., Fernandez-Capetillo, O., Chen, H. T., Reina San Martin, B. Van Laethem, $F$, et al (2005). Role of Nbs1 in the activation of the ATM kinase revealed in humanized mouse models. Nat Cell Biol 7, 675-685.

Doil, C., Mailand, N., Bekker-Jensen, S., Menard, P., Larsen, D. H., Pepperkok, R., et al. (2009). RNF168 binds and amplifies ubiquitin conjugates on damaged chromosomes to allow accumulation of repair proteins. Cell 136, 435-446.

Downs, J. A., Allard, S., Jobin-Robitaille, O., Javaheri, A., Auger, A., Bouchard, N., et al (2004). Binding of chromatin-modifying activities to phosphorylated histone H2A at DNA damage sites. Mol Cell 16, 979-990.

Du, Y. C., Gu, S., Zhou, J., Wang, T., Cai, H., Macinnes, M. A., et al. (2006). The dynamic alterations of H2AX complex during DNA repair detected by a proteomic approach reveal the critical roles of $\mathrm{Ca}(2+) /$ calmodulin in the ionizing radiation-induced cell cycle arrest. Mol Cell Proteomics 5, 1033-1044.

Dudley, D. D., Chaudhuri, J., Bassing, C. H., \& Alt, F. W. (2005). Mechanism and control of V(D)J recombination versus class switch recombination: Similarities and differences. Adv Immunol 86, 43-112.

Dupre, A., Boyer-Chatenet, L., \& Gautier, J. (2006). Two-step activation of ATM by DNA and the Mre11-Rad50-Nbs1 complex. Nat Struct Mol Biol 13, 451-457.

Elkon, R., Rashi-Elkeles, S., Lerenthal, Y., Linhart, C., Tenne, T., Amariglio, N., et al. (2005). Dissection of a DNA-damage-induced transcriptional network using a combination of microarrays, RNA interference and computational promoter analysis. Genome Biol 6, R43.

Falck, J., Coates, J., \& Jackson, S. P. (2005). Conserved modes of recruitment of ATM, ATR and DNA-PKcs to sites of DNA damage. Nature 434, 605-611.

Fan, J., Yang, X., Wang, W., Wood, W. H., $3^{\text {rd }}$, Becker, K. G., Gorospe, M., et al. (2002). Global analysis of stress-regulated mRNA turnover by using cDNA arrays. Proc Natl Acad Sci U S A 99, 10611-10616.

Fei, P., \& El-Deiry, W. S. (2003). P53 and radiation responses. Oncogene 22, 5774-5783.

Fierz, B., Chatterjee, C., McGinty, R. K., Bar-Dagan, M., Raleigh, D. P., Muir, T. W., et al (2011). Histone H2B ubiquitylation disrupts local and higher-order chromatin compaction. Nat Chem Biol 7, 113-119.

Francia, S., Michelini, F., Saxena, A., Tang, D., de Hoon, M., Anelli, V., et al. (2012) Site-specific DICER and DROSHA RNA products control the DNA-damage response. Nature 488, 231-235.
Gallinari, P., Di Marco, S., Jones, P., Pallaoro, M., Steinkuhler, C., et al. (2007). HDACs, histone deacetylation and gene transcription: From molecular biology to cancer therapeutics. Cell Res 17, 195-211.

Gasch, A. P., Huang, M., Metzner, S., Botstein, D., Elledge, S. J., \& Brown, P. O. (2001). Genomic expression responses to DNA-damaging agents and the regulatory role of the yeast ATR homolog Mec1p. Mol Biol Cell 12, 2987-3003.

Gonfloni, S. (2010a). DNA damage stress response in germ cells: role of c-Abl and clinical implications. Oncogene 29, 6193-6202.

Gonfloni, S. (2010b). Modulating c-Abl nuclear activity as a strategy to preserve female fertility. Cell Cycle 9, 217-218.

Gonfloni, S., Di Tella, L., Caldarola, S., Cannata, S. M., Klinger, F. G., Di Bartolomeo, C., et al. (2009). Inhibition of the c-Abl-TAp63 pathway protects mouse oocytes from chemotherapy-induced death. Nat Med 15, 1179-1185.

Grewal, S. I., \& Jia, S. (2007). Heterochromatin revisited. Nat Rev Genet 8, 35-46.

Guenther, M. G., Levine, S. S., Boyer, L. A., Jaenisch, R., \& Young, R. A. (2007). A chromatin landmark and transcription initiation at most promoters in human cells. Cell $130,77-88$

Heo, K., Kim, H., Choi, S. H., Choi, J., Kim, K., et al. (2008). FACT-mediated exchange of histone variant $\mathrm{H} 2 \mathrm{AX}$ regulated by phosphorylation of $\mathrm{H} 2 \mathrm{AX}$ and ADP-ribosylation of Spt16. Mol Cell 30, 86-97.

Hofmann, K. (2009). Ubiquitin-binding domains and their role in the DNA damage response. DNA Repair (Amst) 8, 544-556.

Huen, M. S., Grant, R., Manke, I., Minn, K., Yu, X., Yaffe, M. B., et al. (2007). RNF8 transduces the DNA-damage signal via histone ubiquitylation and checkpoint protein assembly. Cell 131, 901-914.

Ikura, T., Tashiro, S., Kakino, A., Shima, H., Jacob, N., Amunugama, R., et al. (2007). DNA damage-dependent acetylation and ubiquitination of H2AX enhances chromatin dynamics. Mol Cell Biol 27, 7028-7040.

Jackson, S. P., \& Bartek, J. (2009). The DNA-damage response in human biology and disease. Nature 461, 1071-1078.

Jakob, B., Splinter, J., Conrad, S., Voss, K. O., Zink, D., Durante, M., et al. (2011). DNA double-strand breaks in heterochromatin elicit fast repair protein recruitment, histone H2AX phosphorylation and relocation to euchromatin. Nucleic Acids Res 39 . 6489-6499.

Jiang, Z., Kamath, R., Jin, S., Balasubramani, M., Pandita, T. K., \& Rajasekaran, B. (2011). Tip60-mediated acetylation activates transcription independent apoptotic activity of Abl. Mol Cancer 10, 88

Jiang, X., Sun, Y., Chen, S., Roy, K., \& Price, B. D. (2006). The FATC domains of PIKK proteins are functionally equivalent and participate in the Tip60-dependent activation of DNA-PKcs and ATM. J Biol Chem 281, 15741-15746.

Johnson, C. A., White, D. A., Lavender, J. S., O'Neill, L. P., \& Turner, B. M. (2002). Human class I histone deacetylase complexes show enhanced catalytic activity in the presence of ATP and co-immunoprecipitate with the ATP-dependent chaperone protein Hsp70. J Biol Chem 277, 9590-9597.

Keeney, S., \& Neale, M. J. (2006). Initiation of meiotic recombination by formation of DNA double-strand breaks: Mechanism and regulation. Biochem Soc Trans 34, $523-525$.

Keogh, M. C., Kim, J. A., Downey, M., Fillingham, J., Chowdhury, D., Harrison, J. C., et al. (2006). A phosphatase complex that dephosphorylates gammaH2AX regulates DNA damage checkpoint recovery. Nature 439, 497-501.

Kerscher, O., Felberbaum, R., \& Hochstrasser, M. (2006). Modification of proteins by ubiquitin and ubiquitin-like proteins. Annu Rev Cell Dev Biol 22, 159-180.

Kim, J. A., Kruhlak, M., Dotiwala, F., Nussenzweig, A., \& Haber, J. E. (2007). Heterochromatin is refractory to gamma-H2AX modification in yeast and mammals. J Cell Biol $178,209-218$.

Kolas, N. K., Chapman, J. R., Nakada, S., Ylanko, J., Chahwan, R., Sweeney, F. D., et al. (2007). Orchestration of the DNA-damage response by the RNF8 ubiquitin ligase. Science 318, 1637-1640.

Kouzarides, T. (2007). Chromatin modifications and their function. Cell 128, 693-705.

Krebs, J. E. (2007). Moving marks: Dynamic histone modifications in yeast. Mol Biosyst $3,590-597$.

Kryston, T. B., Georgiev, A. B., Pissis, P., \& Georgakilas, A. G. (2011). Role of oxidative stress and DNA damage in human carcinogenesis. Mutat Res 711, 193-201.

Lee, J. H., \& Paull, T. T. (2005). ATM activation by DNA double-strand breaks through the Mre11-Rad50-Nbs1 complex. Science 308, 551-554.

Li, X., Corsa, C. A., Pan, P. W., Wu, L., Ferguson, D., Yu, X., et al. (2010). MOF and H4 K16 acetylation play important roles in DNA damage repair by modulating recruitment of DNA damage repair protein Mdc1. Mol Cell Biol 30, 5335-5347.

Lieber, M. R. (2010). The mechanism of double-strand DNA break repair by the nonhomologous DNA end-joining pathway. Annu Rev Biochem 79, 181-211.

Luijsterburg, M. S., \& van Attikum, H. (2011). Chromatin and the DNA damage response: The cancer connection. Mol Oncol 5, 349-367.

Lukas, J. (2010). The interface between the ubiquitin family and the DNA damage response. EMBO Rep 11, 907-909.

Lukas, J., Lukas, C., \& Bartek, J. (2011). More than just a focus: The chromatin response to DNA damage and its role in genome integrity maintenance. Nat Cell Biol 13, 1161-1169.

Maiani, E., Di Bartolomeo, C., Klinger, F. G., Cannata, S. M., Bernardini, S., Chateauvieux, S., et al. (2012). Reply to: Cisplatin-induced primordial follicle oocyte killing and loss of fertility are not prevented by imatinib. Nat Med 18, 1172-1174

Maiani, E., Diederich, M., \& Gonfloni, S. (2011). DNA damage response: The emerging role of c-Abl as a regulatory switch? Biochem Pharmacol 82, 1269-1276.

Mailand, N., Bekker-Jensen, S., Faustrup, H., Melander, F., Bartek, J., Lukas, C., et al. (2007). RNF8 ubiquitylates histones at DNA double-strand breaks and promotes assembly of repair proteins. Cell 131, 887-900.

Margueron, R., \& Reinberg, D. (2010). Chromatin structure and the inheritance of epigenetic information. Nat Rev Genet 11, 285-296. 
Matsuoka, S., Ballif, B. A., Smogorzewska, A., McDonald, E. R., $3^{\text {rd }}$, Hurov, K. E., \& Luo, J. (2007). ATM and ATR substrate analysis reveals extensive protein networks responsive to DNA damage. Science 316, 1160-1166.

Meltser, V., Ben-Yehoyada, M., \& Shaul, Y. (2011). c-Abl tyrosine kinase in the DNA damage response: Cell death and more. Cell Death Differ 18, 2-4.

Miller, K. M., \& Jackson, S. P. (2012). Histone marks: repairing DNA breaks within the context of chromatin. Biochem Soc Trans 40, 370-376.

Minucci, S., \& Pelicci, P. G. (2006). Histone deacetylase inhibitors and the promise of epigenetic (and more) treatments for cancer. Nat Rev Cancer 6, 38-51.

Mohammad, D. H., \& Yaffe, M. B. (2009). 14-3-3 proteins, FHA domains and BRCT domains in the DNA damage response. DNA Repair (Amst) 8, 1009-1017.

Morrison, A. J., Highland, J., Krogan, N. J., Arbel-Eden, A., Greenblatt, J. F., Haber, J. E., et al. (2004). INO80 and gamma-H2AX interaction links ATP-dependent chromatin remodeling to DNA damage repair. Cell 119, 767-775.

Murr, R., Loizou, J. I., Yang, Y. G., Cuenin, C., Li, H., Wang, Z. Q., et al. (2006). Histone acetylation by Trrap-Tip60 modulates loading of repair proteins and repair of DNA double-strand breaks. Nat Cell Biol 8, 91-99.

Nazarov, I. B., Smirnova, A. N., Krutilina, R. I., Svetlova, M. P., Solovjeva, L. V., Nikiforov, A. A., et al. (2003). Dephosphorylation of histone gamma-H2AX during repair of DNA double-strand breaks in mammalian cells and its inhibition by calyculin A. Radiat Res 160, 309-317.

Negrini, S., Gorgoulis, V. G., \& Halazonetis, T. D. (2010). Genomic instability-An evolving hallmark of cancer. Nat Rev Mol Cell Biol 11, 220-228.

Panier, S., \& Durocher, D. (2009). Regulatory ubiquitylation in response to DNA double-strand breaks. DNA Repair (Amst) 8, 436-443.

Papamichos-Chronakis, M., Krebs, J. E., \& Peterson, C. L. (2006). Interplay between Ino80 and Swr1 chromatin remodeling enzymes regulates cell cycle checkpoint adaptation in response to DNA damage. Genes Dev 20, 2437-2449.

Paulsen, R. D., Soni, D. V., Wollman, R., Hahn, A. T., Yee, M. C., Guan, A., et al. (2009). A genome-wide siRNA screen reveals diverse cellular processes and pathways that mediate genome stability. Mol Cell 35, 228-239.

Pellegrini, M., Celeste, A., Difilippantonio, S., Guo, R., Wang, W., Feigenbaum, L., et al. (2006). Autophosphorylation at serine 1987 is dispensable for murine ATM activation in vivo. Nature $443,222-225$.

Peng, J. C., \& Karpen, G. H. (2008). Epigenetic regulation of heterochromatic DNA stability. Curr Opin Genet Dev 18, 204-211.

Peters, A. H., O'Carroll, D., Scherthan, H., Mechtler, K., Sauer, S., Schofer, C., et al. (2001). Loss of the Suv39h histone methyltransferases impairs mammalian heterochromatin and genome stability. Cell 107, 323-337.

Pokholok, D. K., Harbison, C. T., Levine, S., Cole, M., Hannett, N. M., Lee, T. I., et al. (2005). Genome-wide map of nucleosome acetylation and methylation in yeast. Cell 122, 517-527.

Polo, S. E., \& Jackson, S. P. (2011). Dynamics of DNA damage response proteins at DNA breaks: A focus on protein modifications. Genes Dev 25, 409-433.

Quivy, J. P., Roche, D., Kirschner, D., Tagami, H., Nakatani, Y., \& Almouzni, G. (2004). A CAF-1 dependent pool of HP1 during heterochromatin duplication. EMBO J 23, 3516-3526.

Ramaekers, C. H., \& Wouters, B. G. (2011). Regulatory functions of ubiquitin in diverse DNA damage responses. Curr Mol Med 11, 152-169.

Reinhardt, H. C., Cannell, I. G., Morandell, S., \& Yaffe, M. B. (2011). Is post-transcriptional stabilization, splicing and translation of selective mRNAs a key to the DNA damage response? Cell Cycle 10, 23-27.

Rieger, K. E., \& Chu, G. (2004). Portrait of transcriptional responses to ultraviolet and ionizing radiation in human cells. Nucleic Acids Res 32, 4786-4803.

Roos, W. P., \& Kaina, B. (2012). DNA damage-induced apoptosis: From specific DNA lesions to the DNA damage response and apoptosis. Cancer Lett [Epub ahead of print]. http://www.cancerletters.info/article/S0304-3835(12)00032-8/abstract

Ruthenburg, A. J., Li, H., Patel, D. J., \& Allis, C. D. (2007). Multivalent engagement of chromatin modifications by linked binding modules. Nat Rev Mol Cell Biol 8, 983-994.

Shahbazian, M. D., \& Grunstein, M. (2007). Functions of site-specific histone acetylation and deacetylation. Annu Rev Biochem 76, 75-100.

Shanbhag, N. M., Rafalska-Metcalf, I. U., Balane-Bolivar, C., Janicki, S. M., \& Greenberg, R. A. (2010). ATM-dependent chromatin changes silence transcription in cis to DNA double-strand breaks. Cell 141, 970-981.

Sharma, G. G., So, S., Gupta, A., Kumar, R., Cayrou, C., Avvakumov, N., et al. (2010). MOF and histone $\mathrm{H} 4$ acetylation at lysine 16 are critical for DNA damage response and double-strand break repair. Mol Cell Biol 30, 3582-3595.

Shogren-Knaak, M., \& Peterson, C. L. (2006). Switching on chromatin: Mechanistic role of histone H4-K16 acetylation. Cell Cycle 5, 1361-1365.

Shrivastav, M., De Haro, L. P., \& Nickoloff, J. A. (2008). Regulation of DNA double-strand break repair pathway choice. Cell Res 18, 134-147.

Soria, G., Polo, S. E., \& Almouzni, G. (2012). Prime, repair, restore: The active role of chromatin in the DNA damage response. Mol Cell 46, 722-734.
Stewart, G. S., Panier, S., Townsend, K., Al-Hakim, A. K., Kolas, N. K., \& Miller, E. S. (2009). The RIDDLE syndrome protein mediates a ubiquitin-dependent signaling cascade at sites of DNA damage. Cell 136, 420-434.

Stucki, M., Clapperton, J. A., Mohammad, D., Yaffe, M. B., Smerdon, S. J., \& Jackson, S. P. (2005). MDC1 directly binds phosphorylated histone H2AX to regulate cellular responses to DNA double-strand breaks. Cell 123, 1213-1226.

Suganuma, T., \& Workman, J. L. (2011). Signals and combinatorial functions of histone modifications. Annu Rev Biochem 80, 473-499.

Sun, Y., Jiang, X., Chen, S., Fernandes, N., \& Price, B. D. (2005). A role for the Tip60 histone acetyltransferase in the acetylation and activation of ATM. Proc Natl Acad Sci $U$ S A 102, 13182-13187.

Sun, Y., Jiang, X., Xu, Y., Ayrapetov, M. K., Moreau, L. A., Whetstine, J. R., et al. (2009). Histone $\mathrm{H} 3$ methylation links DNA damage detection to activation of the tumour suppressor Tip60. Nat Cell Biol 11, 1376-1382.

Sun, Y., Jiang, X., \& Price, B. D. (2010). Tip60: connecting chromatin to DNA damage signaling. Cell Cycle 9, 930-936.

Sun, Y., Xu, Y., Roy, K., Price, B. D., et al. (2007). DNA damage-induced acetylation of lysine 3016 of ATM activates ATM kinase activity. Mol Cell Biol 27, 8502-8509.

Svejstrup, J. Q. (2002). Mechanisms of transcription-coupled DNA repair. Nat Rev Mol Cell Biol 3, 21-29.

Szenker, E., Ray-Gallet, D., \& Almouzni, G. (2011). The double face of the histone variant H3.3. Cell Res 21, 421-434.

Takata, M., Sasaki, M. S., Sonoda, E., Morrison, C., Hashimoto, M., Utsumi, H., et al. (1998). Homologous recombination and non-homologous end-joining pathways of DNA double-strand break repair have overlapping roles in the maintenance of chromosomal integrity in vertebrate cells. EMBO J 17, 5497-5508.

Talbert, P. B., \& Henikoff, S. (2010). Histone variants-ancient wrap artists of the epigenome. Nat Rev Mol Cell Biol 11, 264-275.

Ulrich, H. D., \& Walden, H. (2010). Ubiquitin signalling in DNA replication and repair Nat Rev Mol Cell Biol 11, 479-489.

Uziel, T., Lerenthal, Y., Moyal, L., Andegeko, Y., Mittelman, L., \& Shiloh, Y. (2003). Requirement of the MRN complex for ATM activation by DNA damage. EMBO $J$ 22, 5612-5621.

Vakoc, C. R., Sachdeva, M. M., Wang, H., \& Blobel, G. A. (2006). Profile of histone lysine methylation across transcribed mammalian chromatin. Mol Cell Biol 26 9185-9195.

van Attikum, H., Fritsch, O., Hohn, B., \& Gasser, S. M. (2004). Recruitment of the INO80 complex by H2A phosphorylation links ATP-dependent chromatin remodeling with DNA double-strand break repair. Cell 119, 777-788.

van Attikum, H., \& Gasser, S. M. (2005). The histone code at DNA breaks: A guide to repair? Nat Rev Mol Cell Biol 6, 757-765.

van Attikum, H., \& Gasser, S. M. (2009). Crosstalk between histone modifications during the DNA damage response. Trends Cell Biol 19, 207-217.

Vichi, P., Coin, F., Renaud, J. P., Vermeulen, W., Hoeijmakers, J. H., Moras, D., et al. (1997). Cisplatin- and UV-damaged DNA lure the basal transcription factor TFIID/TBP. EMBO J 16, 7444-7456.

Wang, B., \& Elledge, S. J. (2007). Ubc13/Rnf8 ubiquitin ligases control foci formation of the Rap80/Abraxas/Brca1/Brcc36 complex in response to DNA damage. Proc Natl Acad Sci U S A 104, 20759-20763.

Wang, X., Zeng, L., Wang, J., Chau, J. F., Lai, K. P., Jia, D., et al. (2011). A positive role for c-Abl in Atm and Atr activation in DNA damage response. Cell Death Differ 18, 5-15.

Ward, I. M., \& Chen, J. (2001). Histone H2AX is phosphorylated in an ATR-dependent manner in response to replicational stress. J Biol Chem 276, 47759-47762.

Whetstine, J. R., Nottke, A., Lan, F., Huarte, M., Smolikov, S., Chen, Z., et al. (2006). Reversal of histone lysine trimethylation by the JMJD2 family of histone demethylases. Cell 125, 467-481.

Xiao, A., Li, H., Shechter, D., Ahn, S. H., Fabrizio, L. A., Erdjument-Bromage, H., et al. (2009). WSTF regulates the H2A.X DNA damage response via a novel tyrosine kinase activity. Nature 457, 57-62.

Xu, Y., \& Price, B. D. (2011). Chromatin dynamics and the repair of DNA double strand breaks. Cell Cycle 10, 261-267.

Yamagata, K., \& Kitabayashi, I. (2009). Sirt1 physically interacts with Tip60 and negatively regulates Tip60-mediated acetylation of H2AX. Biochem Biophys Res Commun 390, 1355-1360.

Yuan, J., Adamski, R., \& Chen, J. (2010). Focus on histone variant H2AX: To be or not to be. FEBS Lett 584, 3717-3724.

Yuan, J., \& Chen, J. (2010). MRE11-RAD50-NBS1 complex dictates DNA repair independent of H2AX. J Biol Chem 285, 1097-1104.

Yun, M., Wu, J., Workman, J. L., \& Li, B. (2011). Readers of histone modifications. Cell Res $21,564-578$.

Zou, L., \& Elledge, S. J. (2003). Sensing DNA damage through ATRIP recognition of RPA-ssDNA complexes. Science 300, 1542-1548. 